\title{
Improved Equivalent Source Method to Predict Sound Scattered by Solid Surfaces
}

\author{
Chen $\mathrm{Xu}^{1,2 *}$, Yijun $\mathrm{Mao}^{1,2 \dagger}$ and Zhiwei $\mathrm{Hu}^{1 *}$, \\ 1 University of Southampton, SO17 1BJ Southampton, United Kingdom \\ 2 Xi'an Jiaotong University, 710049 Xi'an, People's Republic of China
}

The equivalent source method is an efficient numerical method to predict sound scattered by solid surfaces. Compared with the conventional boundary element method, the equivalent source method has inherent advantages in avoiding the problems of singularity and nonuniqueness of the solution. However, the equivalent source method can form an ill-conditioned matrix for some specific cases, such as thin scattering surfaces, resulting in serious computational errors in these situations. In this paper, an improved equivalent source method is developed, in which an efficient regularization method is proposed to reduce the condition number of the coefficient matrix and the regularization parameter can be easily determined. Numerical test cases validate that the improved method usually can reach a satisfactory computational accuracy for predicting sound scattered by thin structures.

\section{Nomenclature}
$\mathbf{A}=$ original coefficient matrix
$\mathbf{A}^{\prime}=$ regularized coefficient matrix
B = diagonal matrix defined in Eq. (13)
b $=$ column vector
$c_{0} \quad=\quad$ speed of sound $\mathrm{m} \cdot \mathrm{s}^{-1}$
I = unit diagonal matrix
$k=$ wavenumber, $\mathrm{m}^{-1}$

\footnotetext{
*Research Fellow.c.xu@soton.ac.uk; xuchen1983@mail.xjtu.edu.cn.

† Corresponding author. y.mao@soton.ac.uk; maoyijun@mail.xjtu.edu.cn. Senior Member AIAA.

\$ Lecturer. z.hu@soton.ac.uk. Member AIAA.
} 


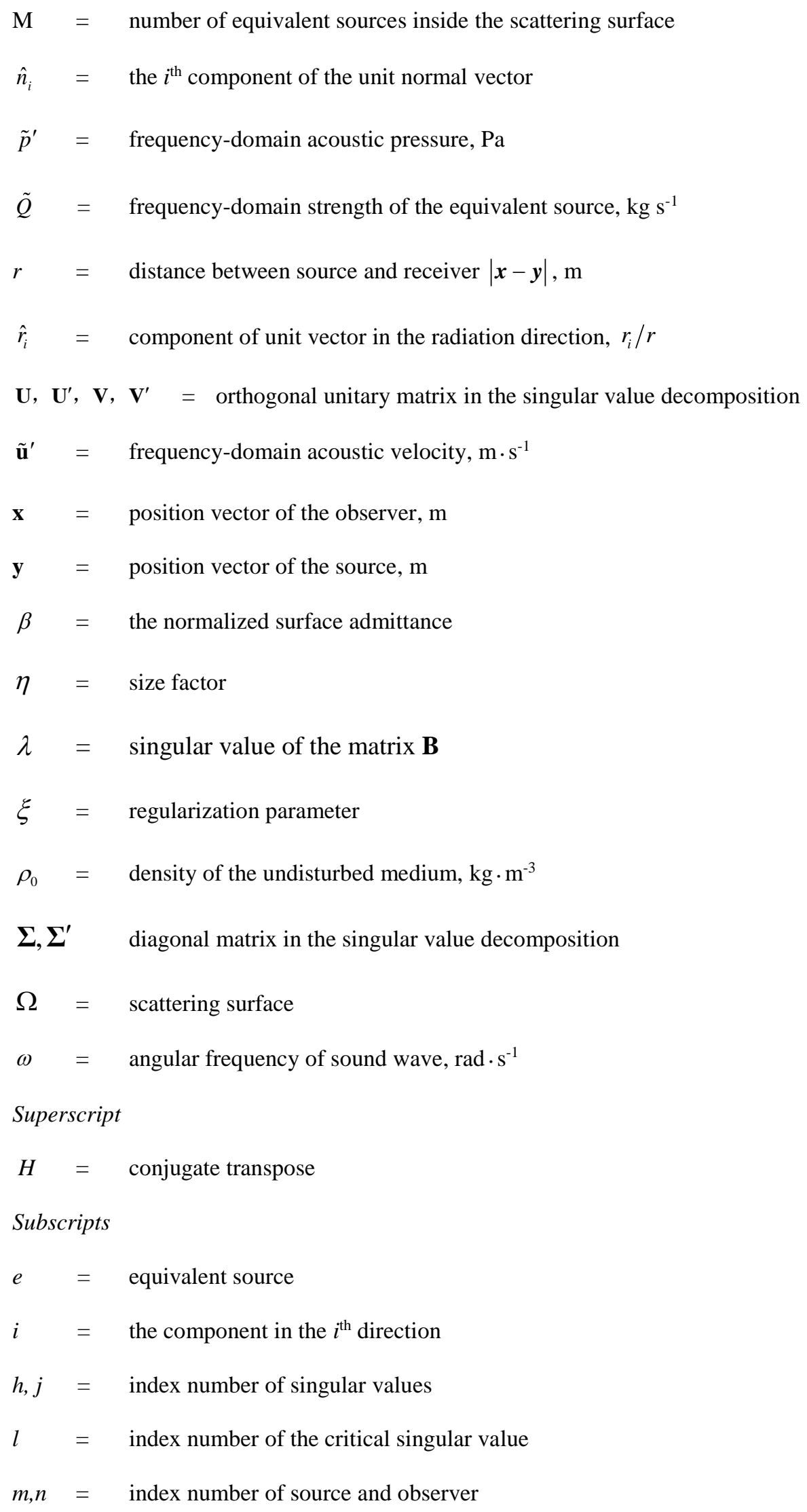




$$
\begin{aligned}
& q=\text { the } q^{\text {th }} \text { iteration of the regularization } \\
& I \quad=\quad \text { incident component } \\
& S \quad=\quad \text { scattering component }
\end{aligned}
$$

\section{Introduction}

W UMERICAL methods developed to predict sound scattered by solid surfaces over past decades can be divided into two catalogues. The first is the differential method, in which the wave equation is discretized temporally and spatially, then solved in the entire computational domain. The second is the integral method, which predicts the acoustic scattering phenomena based on the method of the Green's function. Each method has its advantages and disadvantages, but researchers prefer to use the integral method to predict sound scattered by solid surfaces in a quiescent or uniformly moving acoustic medium owing to the following two reasons. Firstly, the integral method does not introduce a dispersion error which should be carefully treated in the differential method, thus the integral method is easier to achieve a high-accuracy computational result. Secondly, only the scattering surfaces rather than the entire computational domain need to be discretized in the integral method, thus the integral method is more efficient for predicting sound at any receiver positions. In this paper, we employ the integral method to compute numerically sound scattered by solid surfaces.

Boundary element method (BEM) is a commonly-used integral method, and many computational codes have been developed by researchers. The conventional BEM suffers from the singularity and non-uniqueness problems in some specific cases, and various treatment methods have been developed to resolve these issues. However, these treatment methods usually need complicated mathematical techniques and inevitably increase the computational cost. An alternative method is the equivalent source method (ESM), which congenitally avoids the singularity and nonuniqueness problems. Detailed reviews on the recent progress of the ESM can be found in Ref. [1]. However, a drawback of the ESM is that there are no general guidelines to determine the types and positions of the equivalent sources, thus a scaled replica of the scattering surface is usually used to locate the equivalent sources. Moreover, another drawback of the ESM is that it would form an ill-conditioned coefficient matrix in some cases, and this causes a deterioration of the computational accuracy in the ESM. For example, the coefficient matrix is prone to be illconditioned if the distance of adjacent equivalent sources is not large enough compared to the wave length. Therefore, a large ratio between the replica and the scattering surface, such as 0.8-0.9 [2], is usually recommended to avoid the 
above-mentioned problem. This method is usually effective for thick scattering structures, though fails for thin scattering structures. For a thin scattering structure, all the equivalent sources are located in a narrow region, and this usually forms a large condition number of the coefficient matrix which will cause serve computational errors in the ESM [3]. Actually, the same problem, namely thin-shaped breakdown (TSB) [4], also occurs in the conventional BEM. However, $\mathrm{Wu}$ et al. [5] developed an elegant method, in which the original boundary integral equation is combined with its normal gradient, to perfectly resolve the above-mentioned issue encountered in the BEM. Unfortunately, this method is not suitable for the ESM because all the equivalent sources are placed inside rather than on the scattering surfaces. Actually, some researchers have struggled to resolve the TSB problem of the ESM. Hou et al. [6] employed a complex ESM to predict the sound scattered by thin structures, but found that the positions of the equivalent sources should be carefully fixed in the complex space to guarantee a good computational accuracy. Swift et al. [7] kept the equivalent sources to be coincident with the control points on the scattering surface, but this needs an additional treatment for the singularity problem which also exists in the BEM. The key step of the above two methods is to place the equivalent sources in proper locations in order to avoid formulation of an ill-conditioned coefficient matrix. Some other techniques, such as moving sources method [8] and genetic algorithm [9], were also used to optimize the positions of the equivalent sources. However, it is still difficult to find the optimal positions of the equivalent sources in engineering applications so as to avoid the ill-posed problem for scattering surfaces with an arbitrary shape because there is no general criterion to locate the equivalent sources. More discussions on the optimal positions of the equivalent sources can also be found in the recent review by Lee [1].

Therefore, we usually have to find an effective method to solve the ill-conditioned system of equations. Actually, various methods, such as singular value decomposition (SVD) and Tikhonov method, have been developed to solve the ill-conditioned system of equations over the past decades. Related literature reviews on the SVD can be found in references $[10,11]$ and the application of the SVD in the ESM can refer to $[2,12]$. However, an optimal value of the truncation number in the SVD and of the regularization parameter in the Tikhonov method is usually difficult to determine. This paper develops an improved regularization method which makes it easier to determine the optimal value of the regularization parameter and is robust to solve the ill-conditioned system of equations. Furthermore, the above-mentioned improved regularization method is combined with the ESM aiming to improve the computational accuracy for sound scattered by thin structures. 
The remainder of this paper is organized as follows. Section II presents a brief review of the ESM and the classic regularization methods to solve the ill-posed system of equations, and then an efficient regularization method is developed. In Section III, test cases are performed to compare the computational results obtained from different numerical methods with the analytical results, validating the accuracy of the improved equivalent source method. Section IV draws some conclusions. Detailed mathematical derivations on the regularization method are presented in Appendices.

\section{Numerical Methodology}

\section{A. Review of the equivalent source method}

For sound radiated from sources and scattered by solid surfaces, the total sound field is composed of an incident field and a scattered field. The former is calculated directly with the method of Green's function in free space, and the latter can be solved by using either the BEM or the ESM. Details on the time-domain and frequency-domain ESMs can be found in recent references [1, 2, 12-14], here we only present the main computational steps of the frequencydomain ESM.

Step 1. The method of the Green's function is used to calculate the incident acoustic pressure $p_{I}^{\prime}$ and incident acoustic velocity $\mathbf{u}_{I}^{\prime}$ of the sound radiated from sources and received by collocation points $\mathbf{x}$ located on the scattering surface $\Omega$.

Step 2. A size factor $\eta \in(0,1)$ is usually used to generate a smaller replica of the scattering surface and then to fix the equivalent sources $\mathbf{y}_{e}$ on the smaller replica. Either monopole sources or dipole sources can be used to represent the equivalent sources. Here, only the equivalent monopole sources are used for the sake of simplicity description, and the introduction on the equivalent dipole sources can be found in [14]. Therefore, the following equations are used to establish the acoustic response of the $n^{\text {th }}$ observer to the $m^{\text {th }}$ equivalent source.

$$
\begin{gathered}
4 \pi \tilde{p}_{S}^{\prime}\left(\mathbf{x}_{n}, \omega\right)=-\mathrm{i} \omega \frac{\tilde{Q}_{m} e^{\mathrm{i} k r_{m n}}}{r_{m n}} \\
4 \pi \tilde{u}_{S i}^{\prime}\left(\mathbf{x}_{n}, \omega\right)=\frac{\tilde{Q}_{m} \hat{r}_{m n i}\left(1-\mathrm{i} k r_{m n}\right) e^{\mathrm{i} k r_{m n}}}{\rho_{0} r_{m n}^{2}}
\end{gathered}
$$


where $k$ is the acoustic wavenumber; $\rho_{0}$ is the density of unperturbed fluid; $\tilde{Q}_{m}$ is the strength of the $m^{\text {th }}$ equivalent monopole source, which should be solved in Step 3; $r_{m n}=\left|\mathbf{x}_{n}-\mathbf{y}_{\text {em }}\right|$ is the distance between the $n^{\text {th }}$ observer and the $m^{\text {th }}$ equivalent source; $\hat{r}_{m n i}=\left(x_{n i}-y_{e m i}\right) / r_{m n}$ is the component of the normalized radiation vector in the $i^{\text {th }}$ direction. Step 3. The boundary condition on the scattering surface is as follows:

$$
\beta\left(\tilde{p}_{I}^{\prime}+\tilde{p}_{S}^{\prime}\right)+\rho_{0} c_{0}\left(\tilde{u}_{I i}^{\prime}+\tilde{u}_{S i}^{\prime}\right) \cdot \hat{n}_{i}=0
$$

where $\hat{n}_{i}$ represents the component of unit wall normal vector in the $i^{\text {th }}$ direction, $\beta$ is the normalized surface admittance, which is usually a complex number with a positive real part. Especially, for the acoustically rigid condition $\beta=0$, there is

$$
\left(\tilde{u}_{I i}^{\prime}+\tilde{u}_{S i}^{\prime}\right) \cdot \hat{n}_{i}=0
$$

Once the incident acoustic pressure $p_{I}^{\prime}$ and incident acoustic velocity $\mathbf{u}_{I}^{\prime}$ are evaluated, substituting Eqs. (1) and (2) into Eq. (3) yields the following equation:

$$
\mathbf{A Q}=\mathbf{b}
$$

where $\mathbf{A}$ is a coefficient matrix with each element expressed by

$$
A_{m n}=-\mathrm{i} \omega \beta \frac{e^{\mathrm{i} k r_{m n}}}{4 \pi r_{m n}}+\frac{c_{0} \hat{r}_{m n i} \cdot \hat{n}_{i}\left(1-\mathrm{i} k r_{m n}\right) e^{\mathrm{i} k r_{m n}}}{4 \pi r_{m n}^{2}}
$$

where $\mathbf{Q}=\left[\tilde{Q}_{1}, \tilde{Q}_{2}, \ldots \tilde{Q}_{M}\right]^{T}$ is a column vector, and each element $\tilde{Q}_{m}$ represents the unknown strength of the $m^{\text {th }}$ equivalent monopole source; $\mathbf{b}$ is the known vector related to the incident acoustic pressure and acoustic velocity. Moreover, if we assume the number of the equivalent sources is the same as that of the collocation points, $\mathbf{A}$ is an $\mathrm{M} * \mathrm{M}$ coefficient matrix in this situation. However, it is usually unnecessary to use the same numbers of equivalent sources and collocation points. In the ESM, the number of equivalent sources can be much smaller than that of collocation points as demonstrated in references $[2,12]$, which significantly reduces the computational time and is an important advantage of the ESM.

Eq. (5) is solved numerically to obtain the strengths of all equivalent sources. After that, the acoustic pressure and acoustic velocity received by any observers outside the scattering surfaces can be calculated directly. However, the accuracy of the numerical results is highly dependent on the condition number of the matrix $\mathbf{A}$. Eq. (6) indicates that the matrix $\mathbf{A}$ is related to the distance between equivalent sources inside the scattering surface and collocation points 
as well as the wavenumber. Some methods have been developed in previous studies [6-9] to optimize relative position of equivalent sources and collocation points. However, there are no general guidelines to place the equivalent sources, thus it is usually difficult to ensure that the matrix $\mathbf{A}$ is always well-conditioned. Therefore, we need to find a robust method to numerically solve Eq. (5) once the matrix $\mathbf{A}$ is ill-conditioned, and details will be presented in Sections II.B and II.C

\section{B. Classic methods to solve an ill-posed system of equations}

The Tikhonov and SVD methods are usually effective to solve an ill-posed system of equations. Detailed research can be found in $[10,11,15-20]$, and we just give a brief overview of these two methods here.

Tikhonov [21] firstly proposed a method to solve the ill-conditioned matrix problem by introducing a small-value parameter $\xi$ to form the following regularized equation system

$$
\left(\mathbf{A}^{H} \mathbf{A}+\xi \mathbf{I}\right) \mathbf{Q}=\mathbf{A}^{H} \mathbf{b}
$$

where superscript $H$ represents the conjugate transpose. In order to make sure the matrix $\mathbf{A}^{H} \mathbf{A}+\xi \mathbf{I}$ is well conditioned, an appropriate value of the regularization parameter $\xi$ should be chosen. However, it is usually a difficult issue to determine an optimal value of the regularization parameter $\xi$, and details will be discussed at the end of this sub-section.

The SVD of an $\mathbf{M}^{*} \mathrm{M}$ square matrix $\mathbf{A}$ is expressed as

$$
\mathbf{A}=\mathbf{U} \boldsymbol{\Sigma} \mathbf{V}^{H}
$$

where $\mathbf{U}$ and $\mathbf{V}$ are orthogonal unitary matrices, and they have the following features

$$
\mathbf{U U}^{H}=\mathbf{V} \mathbf{V}^{H}=\mathbf{I}
$$

where $\mathbf{I}$ is the unit diagonal matrix. $\boldsymbol{\Sigma}$ is a diagonal matrix containing the real singular values of the matrix $\mathbf{A}$, and the diagonal elements satisfy the following feature:

$$
\sum_{11} \geq \sum_{22} \ldots \geq \sum_{M M} \geq 0
$$

In the SVD method, all singular values not larger than the critical singular value $\sum_{l l}$, where $l$ represents the index number of the critical singular value, are discarded to reduce the condition number of the coefficient matrix, thus one 
should set $\sum_{l l}=\sum_{l+1, l+1}=\ldots \sum_{M M}=0$. The SVD method is easy to perform, thus it was used widely in previous studies on the ESM to find a least-squares solution $[2,12,14]$.

However, the difficulty of the above two methods is to determine the optimal value of the regularization parameters, i.e., $\xi$ in the Tikhonov method and $l$ in the SVD method. An L-curve method was proposed in the previous study to determine the optimal value of the regularization parameter $\xi$ [17], but investigators have to try many different values of the regularization parameter $\xi$ to find the corner point of the L-curve, and this process will be time-consuming for a high-order matrix. Moreover, in engineering applications, the L-curve sometimes has a round corner which causes that it is difficult to determine the optimal value of the regularization parameter $\xi$. Moreover, in real engineering applications, we cannot ensure that the numerical results can achieve a good computational accuracy because the condition number of the regularized matrix is not always small enough even the above methods are used, see the example shown in [3].

\section{Improved method to solve an ill-conditioned system of equations}

A combined SVD and Tikhonov method was initially proposed by Rothwell [22] and is further developed in this paper to solve an ill-conditioned system. By substituting Eq. (8) into Eq. (7), we can obtain that

$$
\left(\mathbf{V} \boldsymbol{\Sigma} \mathbf{U}^{H} \mathbf{U} \boldsymbol{\Sigma} \mathbf{V}^{H}+\xi \mathbf{I}\right) \mathbf{Q}=\mathbf{V} \boldsymbol{\Sigma} \mathbf{U}^{H} \mathbf{b}
$$

By using Eq. (9) and using the property of a full-rank square matrix, Eq. (11) can be reduced to

$$
\mathbf{U}\left(\boldsymbol{\Sigma}+\xi \boldsymbol{\Sigma}^{-1}\right) \mathbf{V}^{H} \mathbf{Q}=\mathbf{b}
$$

We define the matrix $\mathbf{B}$ as follows

$$
\mathbf{B}=\left[\begin{array}{cccc}
\sum_{11}+\xi / \Sigma_{11} & & & 0 \\
& \sum_{22}+\xi / \Sigma_{22} & & \\
& & \ddots & \\
0 & & & \sum_{M M}+\xi / \Sigma_{M M}
\end{array}\right]
$$

and the condition number of this matrix is

$$
\operatorname{cond}(\mathbf{B})=\frac{\max \left(\sum_{j j}+\xi / \sum_{j j}\right)}{\min \left(\sum_{j j}+\xi / \sum_{j j}\right)}, 1 \leq j \leq M
$$

Therefore, Eq. (12) can be expressed by 


$$
\mathbf{A}^{\prime} \mathbf{Q}=\mathbf{b}
$$

with $\mathbf{A}^{\prime}=\mathbf{U B V} \mathbf{V}^{H}$. Here, we emphasize that $\mathbf{U B V} \mathbf{V}^{H}$ is not the SVD of the matrix $\mathbf{A}^{\prime}$ because the diagonal elements of the matrix $\mathbf{B}$ do not satisfy the following feature:

$$
B_{11} \geq B_{22} \ldots \geq B_{M M} \geq 0
$$

In Ref. [22], the above regularization method was proposed to reduce the condition number of the regularization matrix, but no guidelines were provided to determine the value of $\xi$. In this paper, in order to address the above issue, we present an easy method to determine the value of $\xi$ and to compute the condition number of the regularized matrix. Although $\mathbf{U B V}^{H}$ is the not the SVD of the matrix $\mathbf{A}^{\prime}$, we can find that all the singular values of the matrix $\mathbf{B}$ are the same as those of matrix $\mathbf{A}^{\prime}$. Detailed mathematic proof is given in Appendix A.

If the singular values of the original matrix $\mathbf{A}$ gradually reduce and the regularization parameter is $\xi=\sum_{11} \sum_{M M}$, the condition numbers of the regularized matrix $\mathbf{B}$ and the original matrix $\mathbf{A}$ have the following relationship

$$
\mathrm{CN}(\mathbf{B}) \approx 0.5 \sqrt{\mathrm{CN}(\mathbf{A})}
$$

Detailed derivation can be found in Appendix B. Therefore, this regularization method usually can ensure that the condition number of the regularized matrix is usually much smaller than that of the original matrix. It should be noted that $\xi=\sum_{11} \sum_{M M}$ is a sufficient but not a necessary condition to ensure the condition number of the matrix $\mathbf{B}$ is smaller than that of the matrix $\mathbf{A}$. In real calculations, we can firstly set $\sum_{11} \sum_{M M}$ as the initial value of the regularization parameter $\xi$, then find its optimal value to achieve the minimum condition number of the matrix $\mathbf{B}$. However, we can find that $\sum_{11} \sum_{M M}$ is actually the optimal value of the regularization parameter, and details will be presented in Section III. Moreover, we can find that Eq. (12) is very similar to Eq. (5), thus the above-mentioned regularization method can be used to further reduce the condition number of the regularized matrix $\mathbf{U}\left(\boldsymbol{\Sigma}+\xi \boldsymbol{\Sigma}^{-1}\right) \mathbf{V}^{H}$, and this forms an iterative regularization process. It should be emphasized that the coefficient matrices $\mathbf{A}$ and $\mathbf{B}$ must be square matrices in the above derivation, thus the number of the equivalent sources inside the scattering surface should be equal to that of the collocation points. This is a drawback of the present method 
compared to the conventional ESM, in which the number of the equivalent sources could be less than that of the collocation points $[2,12]$.

\section{Numerical test cases}

\section{A. Sound scattered by a rigid sphere}

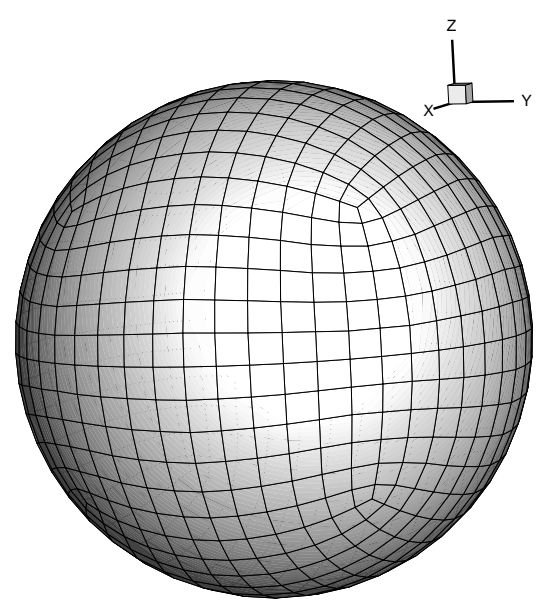

Fig. 1 The mesh of the scattering spherical surface

Incident waves radiate from a stationary monopole point source, the source strength is $1 \mathrm{~kg} / \mathrm{s}$, the wavenumber is $\pi$ and the source is located at $(2 \mathrm{~m}, 0,0)$. The scattering structure is a rigid sphere with a radius of $1 \mathrm{~m}$, and its center is located at the origin of the coordinate system. 60 observers are uniformly located in the XY plane and at a circle with the radius of $20 \mathrm{~m}$. As shown in Fig. 1, the scattering spherical surface is discretized into 864 elements which nearly have the same area.

A small replica of the spherical surface is used to locate the equivalent sources, and the size factor $\eta$ means the ratio of length scales between the small replica and the original scattering surface. Usually, a relative large value of $\eta=0.6-0.9$ is suggested to avoid an ill-conditioned coefficient matrix $\mathbf{A}[2,14]$. However, $\eta=0.2$ is used here to highlight the issue of the ill-conditioned matrix $\mathbf{A}$, as shown in Table 1. On the other hand, when the regularization method and the regularization parameter mentioned in Section II.C are used, Fig. 2 illustrates the variation of the condition number of the regularized matrix with the regularization parameter. The condition number of the regularized 
matrix reaches the minimum when the regularization parameter is equal to the recommended value $\xi=\sum_{11} \sum_{M M}$. In this situation, Table 1 shows that the condition number of the regularized matrices will be greatly reduced, and the condition number of the regularized matrices satisfies the relationship shown in Eq. (17). Similar features can also be observed in other test cases, thus the optimal regularization parameter $\xi=\sum_{11} \sum_{M M}$ is used in this paper to calculate the sound scattered by the solid surface.

Table 1 Comparison of the condition number $(\mathrm{CN})$

\begin{tabular}{lccc}
\hline & CN of matrix $\mathbf{A}$ & $\xi=\sum_{11} \sum_{M M}$ & CN of matrix B \\
\hline Equivalent monopole sources & $2.21 \times 10^{13}$ & $0.24 \times 10^{-4}$ & $1.99 \times 10^{6}$ \\
Equivalent dipole sources & $2.63 \times 10^{13}$ & $1.65 \times 10^{-10}$ & $2.55 \times 10^{6}$ \\
\hline
\end{tabular}

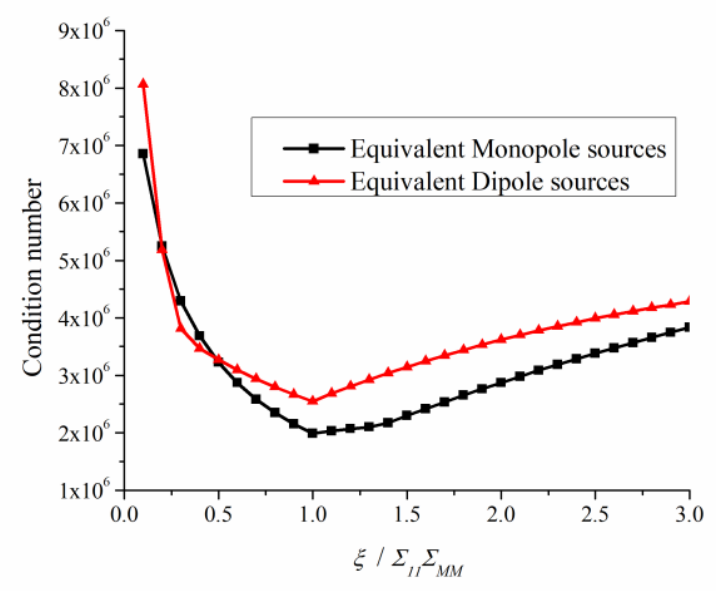

Fig. 2 Variation of the condition number of the regularized matrix with the regularization parameter $\xi$

In this paper, the method of numerically solving the original equation (5) without using a regularization method is named conventional ESM, and the improved ESM corresponds to numerically solving the regularized equation (12). Fig. 3 plots the directivity pattern of the total acoustic pressure, and the numerical results obtained from the conventional ESM suffers from significant computational errors while the numerical results from the improved ESM are consistent with the analytical result. The present test case also indicates that the conventional ESM should carefully select the value of the size factor $\eta$ in order to alleviate the ill-conditioned problem while the improved ESM can retain a good computational accuracy in a wider range of the size factor. 


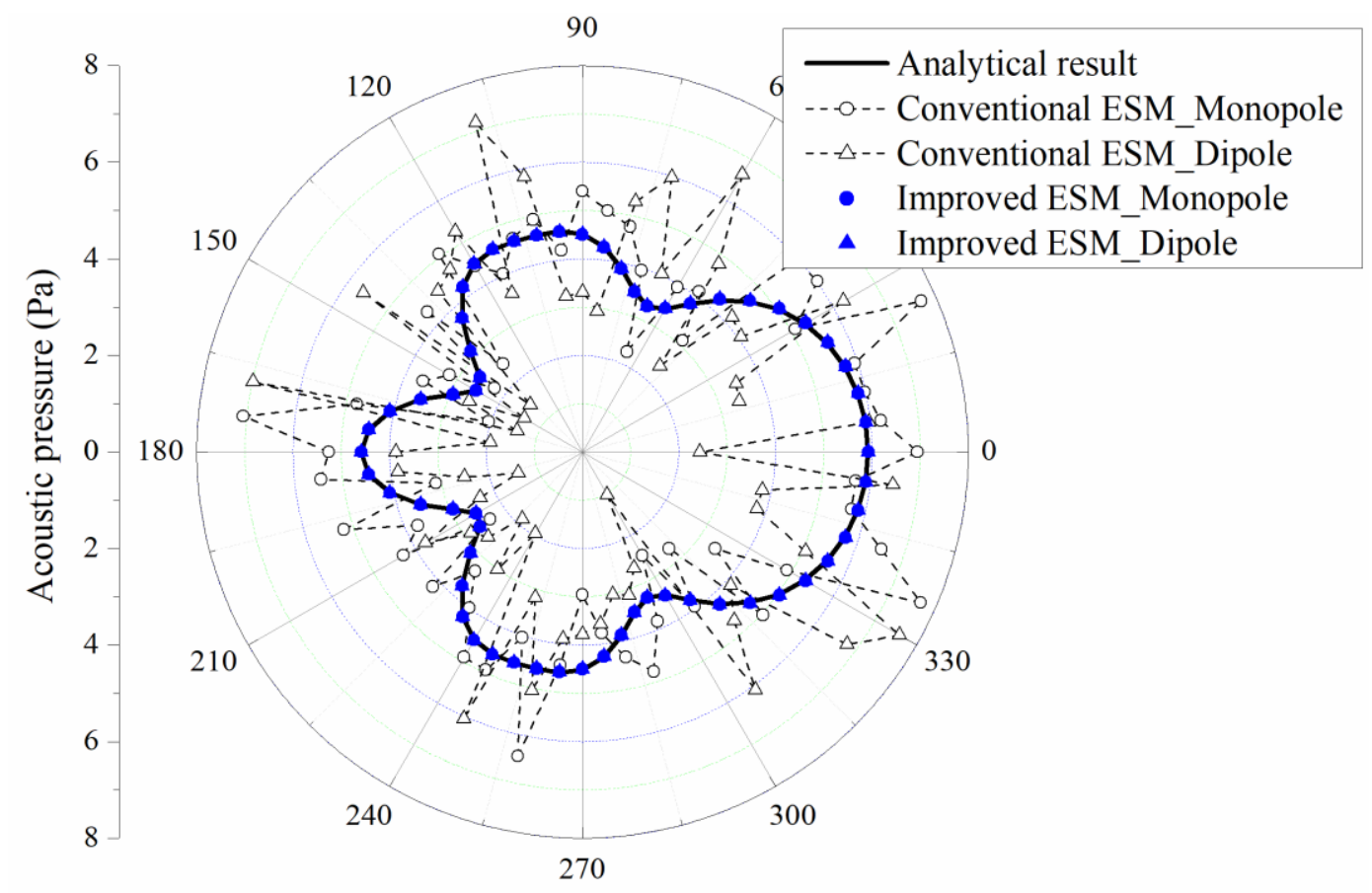

Fig. 3 Directivity pattern of the total acoustic pressure

\section{B. Sound scattered by a thin structure}

Table 2 Geometrical parameters of different oblate spheroids

\begin{tabular}{ccc}
\hline$a / b$ & $a(m)$ & $b(m)$ \\
\hline 2 & 1 & 0.5 \\
8 & 1 & 0.125 \\
1024 & 1 & $9.8 * 10^{-4}$ \\
\hline
\end{tabular}

A plane wave scattered by an oblate spheroid is studied here because the numerical results can be compared with the analytical results to validate the computational accuracy of the improved ESM. The plane wave propagates along the positive $\mathrm{z}$ axis and its wavenumber is 1 . The center of the oblate spheroid is located at the origin of the coordinate system. As shown in Fig. 4, the semi-major axis $a$ and semi-minor axis $b$ of the oblate spheroid are normal to and parallel to $\mathrm{z}$ axis, respectively. Similar to the test cases performed in [3], different shapes of the oblate spheroids are tested, and the related parameters are listed in Table 2. 


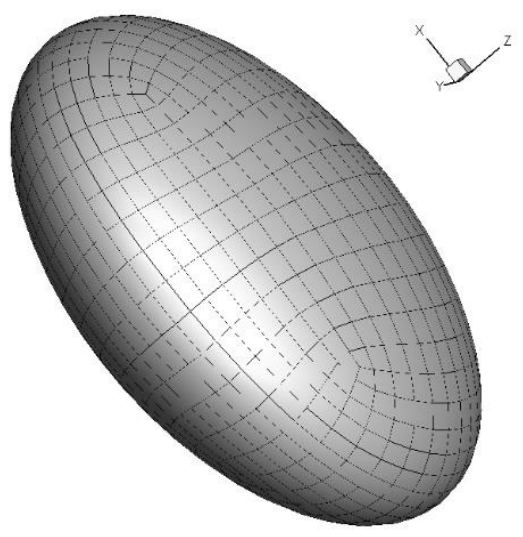

(a)

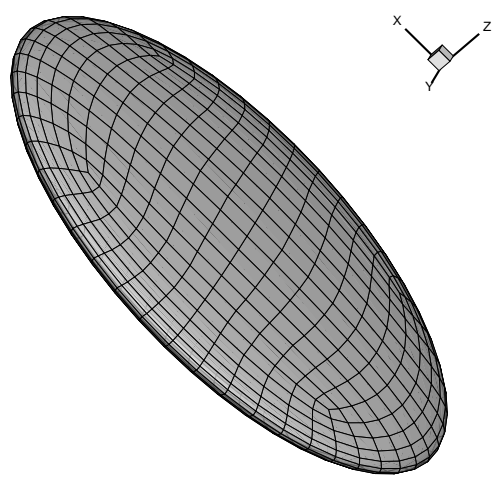

(b)

Fig. 4 The mesh of the scattering oblate spheroids: (a) $a / b=2$; (b) $a / b=8$

Table 3 Condition number and regularization parameter in the case of $a / b=2$

\begin{tabular}{ccc}
\hline & Equivalent monopole sources & Equivalent dipole sources \\
\hline CN of matrix A & $4.0^{*} 10^{7}$ & $3.98 * 10^{6}$ \\
$\xi$ & 41.01 & 0.011 \\
CN of matrix B & 3156.5 & 441.5 \\
\hline
\end{tabular}

Since all the oblate spheroids are similar in shape, the surface elements on each oblate spheroid are generated by transforming from those on a spherical sphere whose radius is equal to the semi-major axis $a$ of the oblate spheroid, in which the $z$ coordinate of the element nodes on the spherical sphere is divided by the coefficient $a / b$ while the $x$ and $y$ coordinates keep invariant in the space transformation. Schematics of the surface mesh for the oblate spheroids with $a / b=2$ and $a / b=8$ are illustrated in Fig. 4 . A recommended value $\eta=0.8$ is used here according to the previous numerical studies on the sound scattered by a sphere. 60 observers are uniformly located in the XZ plane and at a circle with the radius of $3 \mathrm{~m}$.

In the case of $a / b=2$, Table 3 shows that the condition number of the original matrix $\mathbf{A}$ is very large, thus the numerical results calculated from either the monopole equivalent sources or the dipole equivalent sources have a great deviation from the analytical solution, as illustrated in Fig. 5. The condition number of the regularized matrix $\mathbf{B}$ are greatly reduced by using the regularization method developed in Section II.C, and the numerical results obtained from 
the improved ESM are in good agreement with the analytical result. Therefore, this test case illustrates that the improved ESM has the potential to accurately predict sound scattered by a thin structure.

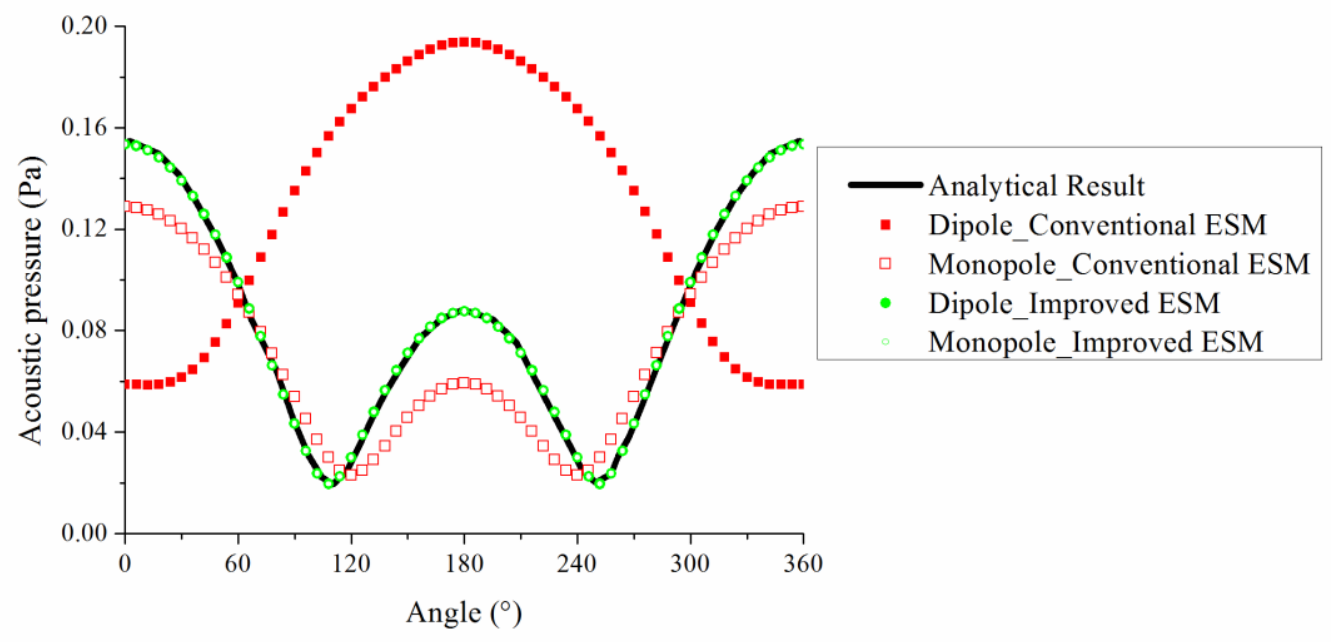

Fig. 5 Directivity pattern of the scattered acoustic pressure at the case of $a / b=2$

Moreover, we consider a thinner scattering structure of $a / b=8$. The previous study of Cheng et al. [3] showed that, even the SVD method was used to solve the system of equations, the numerical result obtained from the ESM still had significant numerical errors in this case. As shown in Table 4, the original matrix $\mathbf{A}$ is seriously ill-conditioned, thus the regularization method combined with the iterative algorithm is used to reduce the condition number of the regularized matrix. $\xi_{q}$ and $\mathbf{B}_{q}$ in Table 4 are the regularization parameter and regularized matrix for the $q^{\text {th }}$ iteration. Fig. 6 compares the numerical results with the analytical solution. Before regularization, the condition number of the original matrix $\mathbf{A}$ is very large and the numerical result significantly deviates from the analytical result. After one round of regularization, the condition number of the regularized matrix $\mathbf{B}_{1}$ is smaller than that of the original matrix A but it is still very large, this improves the numerical result but the numerical errors are still very large. If two rounds of regularizations are performed, the regularized matrix $\mathbf{B}_{2}$ is well-conditioned, and the numerical result is now in reasonable agreement with the analytical result.

Additionally, in order to reach an accurate numerical result, the regularization process not only aims to reduce the condition number of the regularized matrix but also requires that the regularization parameter should be as small as 
possible. Table 4 shows that the regularization parameter $\xi_{2}$ for the equivalent monopole sources is much larger than that for the equivalent dipole sources. Therefore, the numerical errors obtained from the equivalent monopole sources are also larger than those obtained from the equivalent dipole sources, as depicted in Fig. 6.

Table 4 Condition number and regularization parameter in the case of $a / b=8$

\begin{tabular}{ccc}
\hline & Equivalent monopole sources & Equivalent dipole sources \\
\hline CN of matrix $\mathbf{A}$ & $3.15^{*} 10^{12}$ & $4.40^{*} 10^{12}$ \\
$\xi_{1}$ & $7.78^{*} 10^{-4}$ & $2.66^{*} 10^{-6}$ \\
$\mathrm{CN}$ of matrix $\mathbf{B}_{1}$ & $0.89 * 10^{6}$ & $1.05^{*} 10^{6}$ \\
$\xi_{2}$ & 2760.24 & 11.16 \\
$\mathrm{CN}$ of matrix $\mathbf{B}_{2}$ & & 512.1 \\
\hline
\end{tabular}

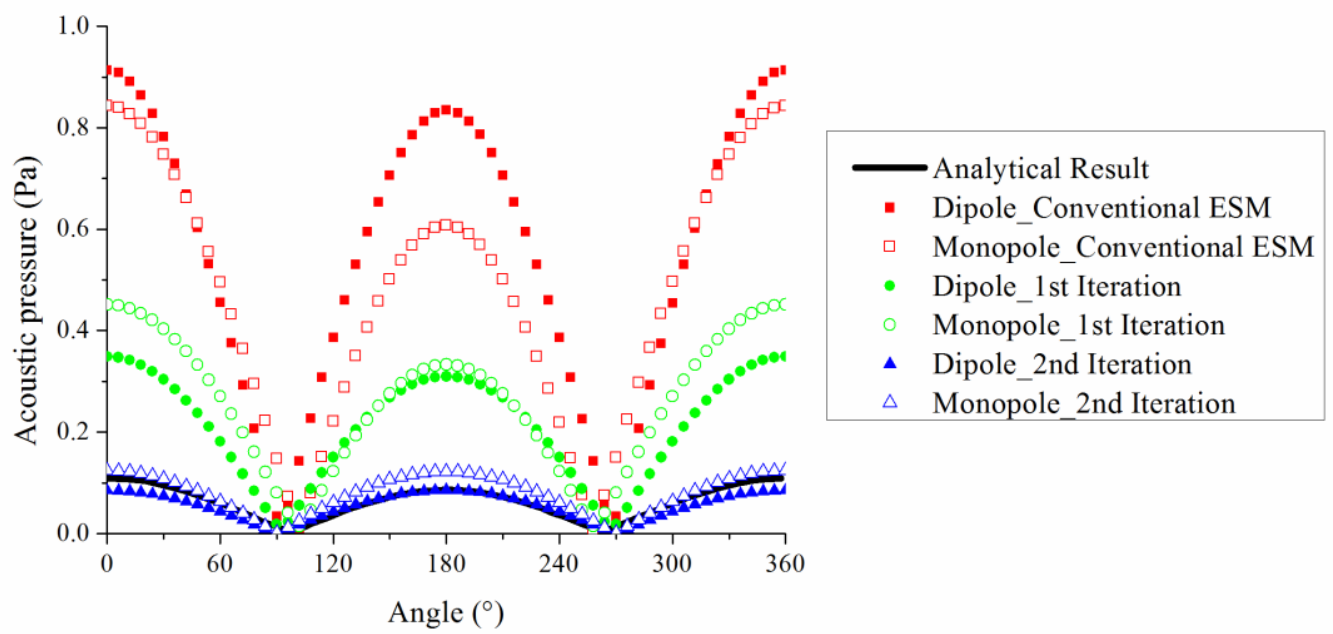

Fig. 6 Directivity pattern of the scattered acoustic pressure at the case of $a / b=8$

The present test case reveals that the iterative algorithm is beneficial to reduce the condition number of the regularized matrix, thus it can be used to reduce the numerical error for sound scattered by a thin structure and to reach a better numerical result compared to the previous study [3]. However, in order to reach an accurate numerical result, the condition number of the regularized matrix and the value of the regularization parameter should be balanced. More discussions on this topic will be performed in the next test case. 
Finally, a very thin oblate spheroid with $a / b=1024$ is considered. Similar to the case of $a / b=8$, the condition number of the original matrix $\mathbf{A}$ is very large, thus two rounds of iterations are performed to reduce the condition number of the regularized matrix, as displayed in Table 5 . However, we can find that the final regularization parameter $\xi_{2}$ is very large, especially for dipole sources, in this test case, which implies that the regularization process has greatly changed the feature of the original coefficient matrix. As analyzed in the previous test case, the condition number of the regularized matrix becomes much smaller but a large regularization parameter would still cause large numerical errors, thus Fig. 7 depicts that the numerical results for both iterations still have serious deviations from the analytical result. This present test case indicates that the improved ESM still cannot be suitable for a very thin scattering surface. Actually, the conventional BEM is also invalid for a very thin scattering surface, and a thin-body BEM [23] is usually recommended in this situation.

Table 5 Condition number and regularization parameter in the case of $a / b=1024$

\begin{tabular}{ccc}
\hline & Equivalent monopole sources & Equivalent dipole sources \\
\hline CN of matrix A & $3.45^{*} 10^{13}$ & $4.33^{*} 10^{13}$ \\
$\xi_{1}$ & $2.50^{*} 10^{-4}$ & $4.05^{*} 10^{-3}$ \\
$\mathrm{CN}$ of matrix $\mathbf{B}_{1}$ & $2.94 * 10^{6}$ & $3.29 * 10^{6}$ \\
$\xi_{2}$ & 2938.0 & $5.33^{*} 10^{4}$ \\
$\mathrm{CN}$ of matrix $\mathbf{B}_{2}$ & & 906.7 \\
\hline
\end{tabular}




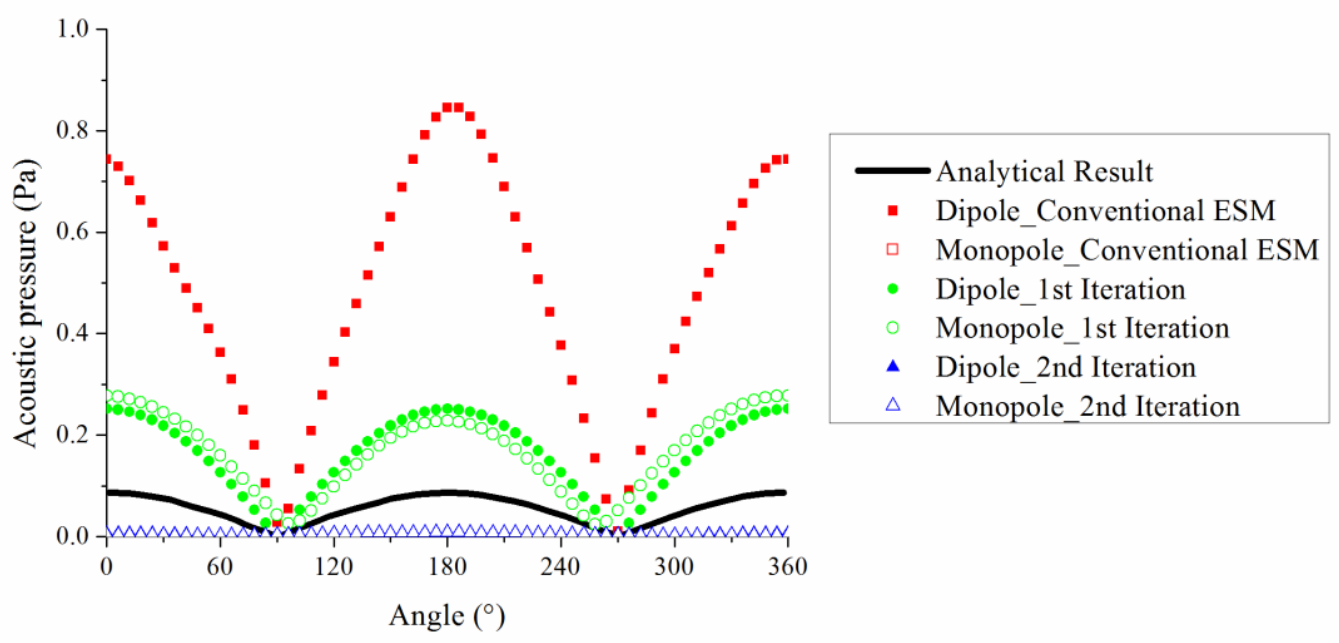

Fig. 7 Directivity pattern of the scattered acoustic pressure at the case of $a / b=1024$

\section{Conclusion}

The ESM can avoid the problems of singular and non-unique solutions that can occur in the BEM, but has the drawback that an ill-conditioned coefficient matrix is possibly formed in some specific cases, such as when denselyspaced equivalent sources are fixed inside a thin scattering structure. In this paper, an efficient method for regularizing the ill-conditioned coefficient matrix, which combines the Tikhonov method with the SVD method, is employed to improve the computational accuracy of the ESM. Compared with the previous regularization methods, the advantage of the present regularization method is that both the optimal value of the regularization parameter and the condition number of the regularized matrix can be easily estimated with the relations $\xi=\sum_{11} \sum_{M M}$ and $\mathrm{CN}(\mathbf{B}) \approx 0.5 \sqrt{\mathrm{CN}(\mathbf{A})}$, respectively. Moreover, an iterative algorithm can be used to further reduce the condition number of the regularized matrix.

Numerical test cases illustrate that the ESM combined with the developed regularization method usually can improve the computational accuracy for sound scattered by a thin structure (e.g., the case of $a / b=2$ ). In particular, the iterative algorithm can be used to further reduce the condition number if the original coefficient matrix is seriously ill-conditioned (e.g., the case of $a / b=8$ ). However, multiple rounds of iterations cause a gradual increase in the regularization parameter. Therefore, in order to reach an accurate numerical result, the condition number of the regularized matrix and the value of the regularization parameter should be balanced. Owing to the above limitation, 
the improved ESM still cannot be suitable for accurately predicting sound scattered by a very thin structure (e.g., the case of $a / b=1024)$. From the viewpoint of practical engineering applications, the improved ESM is beneficial to improve the computational accuracy for sound scattered by airframes or aero-engine nacelles, and a detailed study on its engineering applications will be presented in future.

\section{Appendix A: Singular values of matrices $\mathbf{B}$ and $\mathbf{A}^{\prime}$}

Because $\mathbf{B}$ is a diagonal matrix, we can easily prove that its singular values are $B_{j j}=\sum_{j j}+\xi / \sum_{j j}, j=1,2, \ldots, M$ by using the definition of $\operatorname{det}(\mathbf{B}-\lambda \mathbf{I})=0$, where $\lambda$ is the singular value of the matrix $\mathbf{B}$. Furthermore, the matrix B can be expressed as follows by using the SVD:

$$
\mathbf{B}=\mathbf{U}^{\prime} \boldsymbol{\Sigma}^{\prime} \mathbf{V}^{\prime H}
$$

where both $\mathbf{U}^{\prime}$ and $\mathbf{V}^{\prime}$ are orthogonal unitary matrices. All the singular values of the diagonal matrix $\Sigma^{\prime}$ are same as those of the matrix $\mathbf{B}$, but the diagonal elements of the matrix $\boldsymbol{\Sigma}^{\prime}$ satisfy the following feature:

$$
\sum_{11}^{\prime} \geq \sum_{22}^{\prime} \ldots \geq \sum_{M M}^{\prime}>0
$$

Therefore, the matrix $\mathbf{A}^{\prime}$ can be expressed by

$$
\mathbf{A}^{\prime}=\mathbf{U}^{\prime} \mathbf{\Sigma}^{\prime} \mathbf{V}^{\prime H} \mathbf{V}^{H}
$$

Because $\mathbf{U}, \mathbf{U}^{\prime}, \mathbf{V}$ and $\mathbf{V}^{\prime}$ are unitary matrices, we can easily obtain the following identities:

$$
\begin{gathered}
\mathbf{U}^{\prime}\left(\mathbf{U} \mathbf{U}^{\prime}\right)^{H}=\mathbf{U} \mathbf{U}^{\prime} \mathbf{U}^{\prime H} \mathbf{U}^{H}=\mathbf{I} \\
\mathbf{V}^{\prime H} \mathbf{V}^{H}\left(\mathbf{V}^{\prime H} \mathbf{V}^{H}\right)^{H}=\mathbf{V}^{\prime H} \mathbf{V}^{H} \mathbf{V} \mathbf{V}^{\prime}=\mathbf{I}
\end{gathered}
$$

Equations (A.4) and (A.5) indicate that both the matrices $\mathbf{U} \mathbf{U}^{\prime}$ and $\mathbf{V}^{\prime H} \mathbf{V}^{H}$ are unitary matrices, thus matrix $\mathbf{A}^{\prime}$ is actually decomposed into the following three matrices $\mathbf{U} \mathbf{U}^{\prime}, \boldsymbol{\Sigma}^{\prime}$ and $\mathbf{V}^{\prime H} \mathbf{V}^{H}$ by using the SVD. Based on the above derivation, we can find that matrices $\mathbf{A}^{\prime}, \mathbf{B}$ and $\boldsymbol{\Sigma}^{\prime}$ have the same singular values, thus these three matrices should also have the same the condition number.

\section{Appendix B: Comparison of the condition numbers between $\mathrm{A}^{\prime}$ and $\mathrm{A}$}

The original equation (5) is very similar to the regularized equation (15) except that they have different coefficient matrices. Therefore, we aim to ensure that the condition number of the matrix $\mathbf{A}^{\prime}$ is smaller than the matrix $\mathbf{A}$ by 
selecting an appropriate value of the regularization parameter $\xi$, and this actually requires that the following condition should be satisfied for any index numbers $i$ and $j$ :

$$
\frac{\sum_{h h}+\xi / \sum_{h h}}{\sum_{j j}+\xi / \sum_{j j}}<\frac{\sum_{11}}{\sum_{M M}}=\mathrm{CN}(\mathbf{A})
$$

After some mathematical manipulations, we can obtain the following inequality:

$$
\sum_{h h} \sum_{M M}-\sum_{j j} \Sigma_{11}<\xi\left(\frac{\sum_{11}}{\sum_{j j}}-\frac{\sum_{M M}}{\sum_{h h}}\right)
$$

In order to ensure the above inequality is always valid, $\xi$ must be positive, so we have

$$
\frac{\sum_{h h} \sum_{M M}-\sum_{j j} \sum_{11}}{\xi}<\frac{\sum_{11}}{\sum_{j j}}-\frac{\sum_{M M}}{\sum_{h h}}
$$

Furthermore, if the regularization parameter satisfies

$$
\xi=\sum_{11} \Sigma_{M M}
$$

Inequality (B.3) becomes

$$
\frac{\sum_{h h}}{\sum_{11}}-\frac{\sum_{j j}}{\sum_{M M}}<\frac{\sum_{11}}{\sum_{j j}}-\frac{\sum_{M M}}{\sum_{h h}}
$$

By using the feature given in (10), we can easily prove this inequality is always valid. Moreover, by using Eq. (B.4) the diagonal element of the matrix $\mathbf{B}$ can be expressed by

$$
B_{j j}=\sum_{j j}+\sum_{11} \Sigma_{M M} / \Sigma_{j j}
$$

$\sum_{j j}$ does not increase with the index number $h$, thus we can easily deduce the following inequality

$$
\sum_{j j}+\sum_{11} \sum_{M M} / \sum_{j j} \leq \sum_{11}+\sum_{M M}
$$

Inequality (B.7) implies that when $j$ is equal to 1 or $M, B_{j j}$ achieves the following maximum value

$$
B_{j j, \max }=B_{11}=B_{M M}=\sum_{11}+\sum_{M M}
$$

Moreover, Eq. (B.6) indicates that the minimum singular value should satisfy the following inequality

$$
B_{j j, \text { min }} \geq 2 \sqrt{\sum_{11} \sum_{M M}}
$$


where the equation is valid only at $\sum_{j j}=\sqrt{\sum_{11} \sum_{M M}}$. Therefore, the condition number of the regularized matrix is

$$
\mathrm{CN}\left(\mathbf{A}^{\prime}\right)=\mathrm{CN}(\mathbf{B}) \leq \frac{B_{j j, \text { max }}}{B_{j j, \text { min }}}=\frac{\sum_{11}+\sum_{M M}}{2 \sqrt{\sum_{11} \sum_{M M}}} \approx \frac{1}{2} \sqrt{\frac{\sum_{11}}{\sum_{M M}}}=0.5 \sqrt{\mathrm{CN}(\mathbf{A})}
$$

Eq. (B.10) presents a simple method to estimate the condition number of the regularized matrix

\section{Acknowledgments}

The research has been supported by the Newton Fund (No. IE141516) and the International Postdoctoral Exchange Fellowship Program (No. 20160101). The authors acknowledge anonymous reviewers and the associate editor, Prof. Dimitri Papamoschou, for their valuable comments to improve the quality of the paper.

\section{References}

[1] Lee, S. "Review: The use of equivalent source method in computational acoustics," Journal of Computational Acoustics Vol. 25, No. 01, 2017, p. 1630001.

doi:10.1142/S0218396X16300012

[2] Lee, S., Brentner, K. S., and Morris, P. J. "Assessment of time-domain equivalent source method for acoustic scattering," AIAA Journal Vol. 49, No. 9, 2011, pp. 1897-1906.

doi: $10.2514 / 1 . J 050736$

[3] Cheng, B., Brentner, K., and Morris, P. "Validation of a time-domain equivalent source method for acoustic scattering by an oblate spheroid," AIAA Paper 2013-0759.

doi:10.2514/6.2013-759

[4] Martinez, R. "The thin-shape breakdown (TSB) of the Helmholtz integral equation," The Journal of the Acoustical Society of America Vol. 90, No. 5, 1991, pp. 2728-2738. doi:10.1121/1.401868

[5] $\mathrm{Wu}, \mathrm{T}$. "A direct boundary element method for acoustic radiation and scattering from mixed regular and thin bodies," The Journal of the Acoustical Society of America Vol. 97, No. 1, 1995, pp. 84-91. doi:10.1121/1.412209

[6] Hou, Y., Zhang, X., and Angland, D. "A complex equivalent source method for scattering effect of aircraft noise," AIAA Paper 2014-3302.

doi:10.2514/6.2014-3302

[7] Swift, S., Blaisdell, G., and Lyrintzis, A. "An efficient time-domain equivalent source method for acoustic scattering," International Journal of Aeroacoustics Vol. 14, No. 1, 2015, pp. 133-160.

doi: 10.1260/1475-472X.14.1-2.133 
[8] Kondapalli, P. S., Shippy, D. J., and Fairweather, G. "Analysis of acoustic scattering in fluids and solids by the method of fundamental solutions," The Journal of the Acoustical Society of America Vol. 91, No. 4, 1992, pp. 1844-1854.

doi: $10.1121 / 1.403714$

[9] Gounot, Y. J., and Musafir, R. E. "Genetic algorithms: A global search tool to find optimal equivalent source sets," Journal of Sound and Vibration Vol. 322, No. 1-2, 2009, pp. 282-298.

doi: $10.1016 / j . j s v .2008 .11 .001$

[10] Hansen, P. C., Sekii, T., and Shibahashi, H. "The modified truncated SVD method for regularization in general form," SIAM Journal on Scientific and Statistical Computing Vol. 13, No. 5, 1992, pp. 1142-1150. doi: $10.1137 / 0913066$

[11] Hansen, P. C. "Regularization tools: a Matlab package for analysis and solution of discrete ill-posed problems," Numerical algorithms Vol. 6, No. 1, 1994, pp. 1-35.

doi: 10.1007/BF02149761

[12] Lee, S., Brentner, K. S., and Morris, P. J. "Acoustic scattering in the time domain using an equivalent source method," AIAA Journal Vol. 48, No. 12, 2010, pp. 2772-2780. doi: $10.2514 / 1.45132$

[13] Mark, D., and Ana, T. "Aeroacoustic Scattering via the Equivalent Source Method," AIAA Paper 2004-2937. doi:10.2514/6.2004-2937

[14] Mao, Y. J., Cai, J. C., Gu, Y. Y., and Qi, D. T. "Direct evaluation of acoustic intensity vector field around impedance scattering body," AIAA Journal Vol. 53, No. 5, 2015, pp. 1362-1371.

doi: 10.2514/1.J053431

[15] Reichel, L., and Rodriguez, G. "Old and new parameter choice rules for discrete ill-posed problems," Numerical Algorithms Vol. 63, No. 1, 2013, pp. 65-87. doi: 10.1007/s11075-012-9612-8

[16] Reginska, T. "A regularization parameter in discrete ill-posed problems," SIAM Journal on Scientific Computing Vol. 17, No. 3, 1996, pp. 740-749. doi: $10.1137 / \mathrm{S} 1064827593252672$

[17] Hansen, P. C., and O'Leary, D. P. "The use of the L-curve in the regularization of discrete ill-posed problems," SIAM Journal on Scientific Computing Vol. 14, No. 6, 1993, pp. 1487-1503.

doi: 10.1137/0914086

[18] Hansen, P. C. "Analysis of discrete ill-posed problems by means of the L-curve," SIAM review Vol. 34, No. 4, 1992, pp. 561-580. doi: $10.1137 / 1034115$

[19] Hansen, P. C. "Truncated singular value decomposition solutions to discrete ill-posed problems with illdetermined numerical rank," SIAM Journal on Scientific and Statistical Computing Vol. 11, No. 3, 1990, pp. 503-518.

doi:10.1137/0911028 
[20] Hansen, P. C. "The truncatedsvd as a method for regularization," BIT Numerical Mathematics Vol. 27, No. 4, 1987, pp. 534-553.

doi: 10.1007/BF01937276

[21] Tikhonov, A. N., Arsenin, V. I. A. k., and John, F. Solutions of ill-posed problems, John Wiley, New York, 1977.

[22] Rothwell, E., and Drachman, B. "A unified approach to solving ill-conditioned matrix problems," International Journal for Numerical Methods in Engineering Vol. 28, No. 3, 1989, pp. 609-620. doi: 10.1002/nme.1620280309

[23] $\mathrm{Wu}, \mathrm{T}$. W., and Wan, G. C. "Numerical modeling of acoustic radiation and scattering from thin bodies using a Cauchy principal integral equation," Journal of the Acoustical Society of America Vol. 92, No. 5, 1992, pp. 29002906.

doi: $10.1121 / 1.404375$ 